\title{
Longitudinal Changes in Sexual Risk Behavior Among HIV+ and HIV- Male Injecting Drug Users
}

Curtis Dolezal, Heino F. L. Meyer-Bahlburg, Xinhua Liu, Anke A. Ehrhardt, Theresa M. Exner, Judith G. Rabkin, Jack M. Gorman, Karen Marder \& Yaakov Stern

To cite this article: Curtis Dolezal, Heino F. L. Meyer-Bahlburg, Xinhua Liu, Anke A. Ehrhardt, Theresa M. Exner, Judith G. Rabkin, Jack M. Gorman, Karen Marder \& Yaakov Stern (1999) Longitudinal Changes in Sexual Risk Behavior Among HIV+ and HIV- Male Injecting Drug Users, The American Journal of Drug and Alcohol Abuse, 25:2, 281-303, DOI: 10.1081/ADA-100101861

To link to this article: https://doi.org/10.1081/ADA-100101861

册 Published online: 05 Dec 1999.

Submit your article to this journal $₫$

山 Article views: 46

Q View related articles ¿

Citing articles: 1 View citing articles $ک$ 


\title{
Longitudinal Changes in Sexual Risk Behavior Among HIV+ and HIV- Male Injecting Drug Users
}

\author{
Curtis Dolezal, Ph.D. ${ }^{1}$ \\ Heino F. L. Meyer-Bahlburg, Dr. rer. nat. ${ }^{1, *}$ \\ Xinhua Liu, Ph.D. ${ }^{2}$ \\ Anke A. Ehrhardt, Ph.D. ${ }^{1}$ \\ Theresa M. Exner, Ph.D. ${ }^{1}$ \\ Judith G. Rabkin, Ph.D., M.P.H. ${ }^{1}$ \\ Jack M. Gorman, M.D.' \\ Karen Marder, M.D., M.P.H. ${ }^{1}$ \\ Yaakov Stern, Ph.D. ${ }^{1}$ \\ ${ }^{1}$ HIV Center for Clinical and Behavioral Studies \\ New York State Psychiatric Institute and Columbia University \\ New York, New York \\ ${ }^{2}$ Department of Biostatistics \\ New York State Psychiatric Institute and Columbia University \\ New York, New York
}

\begin{abstract}
Injecting drug users (IDUs) play a prominent role in the transmission of human immunodeficiency virus (HIV), particularly in urban areas such as New York City, where they comprise nearly half of all adult acquired immunodeficiency syndrome (AIDS) cases. Intervention studies have demonstrated that IDUs are responsive to safer sex messages, but sexual behavior appears to be more resistant to change than drug use behavior. This multidisciplinary study (without an intervention component) assesses changes in sexual risk behavior as a function
\end{abstract}

* To whom correspondence should be sent at New York State Psychiatric Institute, 1051 Riverside Drive, Unit 15, New York, NY 10032. Telephone: (212) 543-5299. E-mail: MEYERB@ CHILD.CPMC.COLUMBIA.EDU 
of time, HIV status, and disease progression in a cohort of HIV + and HIV - male IDUs $(N=144)$ for 4 years. Results: For HIV + and HIV - men, there were increases in abstinence and monogamy, with decreases in the frequency of unprotected vaginal/anal sex and sexual risk index scores. With the exception of monogamy, HIV + men reported lower levels of risk. Although there was also a decline in substance use, this accounted for only some of the decline in sexual risk behavior. Among the HIV + men, a CD4 level below 200 was associated with more abstinence and monogamy. HIV-related medical symptoms were associated with increased abstinence, less unprotected sex, and lower sexual risk index scores. Lower neuropsychological memory test scores were associated with increased abstinence and lower sexual risk index scores. Neurological impairment and depression were not associated with sexual risk behavior. Conclusion: IDU men in New York City have modified their sexual behavior toward safer practices. Lower levels of risk are found among HIV + men, particularly those with more progressed HIV illness. Nevertheless, a substantial amount of sexual risk behavior remained in this cohort, indicating the continued need for education and intervention.

This paper focuses on HIV-related sexual risk behaviors among injecting drug users (IDUs) in New York City. While IDUs account for a substantial proportion of acquired immunodeficiency syndrome (AIDS) cases throughout the United States, this is especially true in New York City, where IDUs currently comprise nearly half of adult AIDS diagnoses. Furthermore, over half of New York City's female AIDS cases attributed to heterosexual transmission involve an IDU sex partner, and the vast majority of the city's pediatric AIDS cases have involved a mother who is an IDU (52\%) or the sex partner of an IDU (18\%, with an additional $24 \%$ involving a parent with unidentified risk) (1).

Our study follows a cohort of male IDUs over a 4-year period, beginning in late 1988. At that time, there were an estimated 250,000 IDUs in New York City (2), over half of which were positive for the human immunodeficiency virus (HIV) (3-7). Methadone programs had 29,000 people actively enrolled in 1988 (8), but many more were on waiting lists. Several studies from the late 1980s found high levels of HIV knowledge among IDUs, but also substantial rates of injecting and sexual risk behavior (9).

\section{Reductions in Risk Behavior Among Injecting Drug Users}

Friedman et al. reported less HIV-related risk behavior change among IDUs than among gay men in New York City and suggested that this was partially due to the relative lack of organizations formed by IDUs to provide information and services to their "community" (10). Nevertheless, there are indications that IDUs' practices are changing toward less risky behavior, particularly regarding needle use practices (11-19). Studies that assess sexual behavior among IDUs 
are typically cross-sectional and find that condoms are not used on most sexual occasions (9, 20, 21). Des Jarlais et al. (19) and Booth and Watters (22) reviewed a number of studies that reported modest decreases in sexual risk behavior in response to a variety of interventions. IDUs in those studies were more likely to report changes in injecting behavior than in sexual behavior in every study in which the two were compared. More recent intervention studies have also demonstrated changes toward safer sex $(11,23,24)$.

Some information exists on whether IDUs who are not taking part in an intervention program are modifying their sexual behavior in response to the AIDS epidemic, which is important since formal HIV-related interventions likely reach only a small proportion of IDUs. In surveys of IDUs in New York, approximately half of the participants typically report having changed their sexual behavior toward safer practices (25-27). One study that demonstrates apparent increases in sexual risk behavior is that of Davoli et al. (28), in which two cross-sectional samples of IDUs were compared in Rome, Italy. The only significant difference was that, among those who were HIV - lower rates of condom use with primary partners were reported in 1992 (34\% reported condom use) compared to $1990(46 \%)$.

Cross-sectional surveys and intervention studies (typically involving only a single follow-up assessment) are limited in the extent to which they can address whether changes in sexual risk behavior occur and are maintained over longer periods of time among IDUs who are not in a specific intervention program. There are several reasons to expect sexual risk behavior to decrease over time. First, continued exposure to information about AIDS via television, ad campaigns, health facilities, and drug treatment programs may effect behavior change. The cumulative influence of reinforced safer sex messages might convince individuals that a reduction in risk behavior is advantageous. Already in the mid-1980s, many IDUs in New York City claimed to have received AIDS information from newspapers/magazines (61\%), television $(40 \%)$, or friends (43\%) (13), and information regarding AIDS continues to be widely distributed. Second, as time passes, it becomes more likely that individuals experience the loss of friends or a partner to AIDS or have acquaintances that become HIV positive. These social experiences may increase the salience of the epidemic for the individual. In the study by Selwyn et al. mentioned above (13), 36\% of the IDUs knew someone with AIDS. Loss of a friend or family member to AIDS has been associated with changes in sexual risk behavior among IDU women (29) and gay men (30). In terms of theoretical models designed to explain healthrelated behavior change, increased information and the loss of friends to AIDS should increase the perceived threat of the disease (31; Health Belief Model) and 
increase the perception of risk associated with unsafe drug and sex practices (32; AIDS Risk Reduction Model). Finally, even in the absence of a formal intervention, the procedure itself of assessing sexual behavior may actually influence behavior. Such assessments could increase the participants' interest in HIVrelated issues and increase their awareness of their own risk behavior. In addition, such studies may constitute yet another source of AIDS-related information if pamphlets or service referrals are provided.

\section{Sexual Risk Behavior as a Function of HIV Status and HIV Disease Progression}

Previous studies have found condom use to be more frequent among HIV+ IDUs compared to HIV - IDUs (33-35). Skidmore, Robertson, and Roberts (36) also found that HIV + IDUs showed greater reductions in their number of sexual partners than HIV - participants. There are various possible reasons why lower levels of risk are found among HIV+ IDUs compared to HIV - IDUs. IDUs may modify their behavior fairly soon after receiving a positive HIV test out of a sense of responsibility toward their sexual partners. There may also be changes that occur as a consequence of disease progression. Several factors could contribute to such changes: increased symptoms and feeling ill might reduce a person's interest in sex in general, make it more difficult to find a sex partner, or increase the salience of the disease and thereby motivate the individual to make changes that are subsequently seen as more necessary. Another potential factor is decreasing testosterone with the progression of HIV disease, which may result in diminished sexual desire (37). Yet, an association between disease progression and sexual risk behavior has not clearly been established. A relationship was found between low CD4 counts and lower levels of sexual risk behavior in a cohort of gay men (38), but the association between HIV disease progression and sexual risk behavior remains a largely unexplored area.

HIV disease can take a wide variety of courses and result in various manifestations. As the immune system is compromised, there are often increased symptoms, and many of the systems of the body, including the central and peripheral nervous systems, can be affected. A wide range of neurological manifestations is associated with HIV disease (39-41). In a study involving our sample of IDUs, neurological deficits were more common among HIV + than HIV - men and were associated with lower CD4 levels among the HIV+ men (42). Another report on the sample involved in our analyses (43) focused on neuropsychological performance; it found that, among HIV + men, memory performance was worse 
when the CD4 level fell below 200. Not all studies of IDUs have been able to detect consistent decrements in neuropsychological performance associated with HIV disease, perhaps due to drug-related impairment independent of HIV (4446). However, several studies have demonstrated that impaired memory can be a consequence of HIV infection among gay men and IDUs (47-49).

In terms of psychiatric factors, many researchers have examined whether depression is a common consequence of HIV disease. Studies involving our sample of IDUs $(50,51)$ have shown depressive symptoms to be more common among $\mathrm{HIV}+$ men compared to HIV - men, and that over time, those with fewer symptoms or higher CD4 levels showed more psychological improvement than those with more symptoms or lower CD4 levels. However, recent reviews on the association between depression and HIV disease reveal inconsistent findings (52-54). Similarly, studies assessing the association between depressive symptoms and sexual risk behavior are also inconsistent $(29,55,56)$.

Our study assessed a cohort of HIV + and HIV - male IDUs every 6 months for up to 4 years as part of a multidisciplinary natural history study of HIV disease progression. Based on the information outlined above, we expected reductions in sexual risk behavior over time in both HIV + and HIV - IDU men and lower levels of risk among HIV + men compared to HIV - men. In addition, we wanted to explore whether medical, neurological, neuropsychological, or psychosocial indications of disease progression were associated with lower levels of sexual risk behavior among the HIV + men in our cohort.

\section{METHOD}

\section{Sample}

The current analyses are based on data from a comprehensive natural history study of HIV disease in New York City by the HIV Center for Clinical and Behavioral Studies, modeled after the center's project on homosexual men (57). Preliminary reports covering the baseline (58) and data from years 1 and 2 (59) have been presented previously. This report covers the entire study and uses statistical techniques appropriate for longitudinal data.

All participants were required to know their HIV status prior to study entry and, if HIV+, could not meet 1987 Centers for Disease Control (CDC) criteria for AIDS (60). Other eligibility criteria for IDU men differed slightly between two recruitment sites. Participants recruited through an infectious disease clinic at Harlem Hospital ( $80 \%$ of the sample) had to have used intravenous drugs at 
least 10 times since 1982 and at least once in the previous year. Eligibility requirements for those recruited at the Roosevelt Hospital Drug Treatment Program included attendance at the methadone maintenance program for at least 3 months and the use of intravenous drugs at least 10 times since 1982, but not necessarily in the previous 12 months. Although eligibility criteria were somewhat minimal regarding drug use, recent drug use, including that of heroin, was reported by the vast majority of the sample (51).

Participants were paid $\$ 10$ per hour for lengthy assessments, which included medical, immunologic, neurological, neuropsychological, psychosocial, and psychosexual assessments. The men were seen every 6 months for up to an additional 3.5 years (8 visits). Since psychosexual assessments at baseline inquired about the preceding 6 months, assessments potentially covered a 4-year period.

A convenience sample of 146 IDU men was recruited, and baseline assessments began in late 1988. Of the 146 men, 1 man seroconverted in the course of the study and was excluded from analyses since differences between HIV+ and HIV - men was one of our primary research questions. Another man's baseline psychosexual interview was judged unreliable, and he did not return for later visits. This resulted in a sample of 144 men $(84 \mathrm{HIV}+, 60 \mathrm{HIV}-)$ available for analyses.

\section{Assessment}

Sexual behavior and substance use in conjunction with sex was assessed at each visit by interview using the Sexual Risk Behavior Assessment Schedule, Adult, IDU Men (SERBAS-A-DM), * a somewhat abbreviated version of the SERBAS-A-HOM described in detail elsewhere (61). Participants reported the frequency of various sexual practices with both male partners and female partners (assessed separately). Same-sex partners were relatively uncommon in this cohort. Of the men, 19 had a male partner at some point in the study; only 6 of these reported a male partner on more than one study visit. Even though samesex partners were not particularly common, sexual practices with male partners were considered in each of our sexual risk behavior variables since they also represent potential risk.

The variables analyzed at each visit were (a) abstinence, (b) monogamy, (c)

\footnotetext{
* H. F. L. Meyer-Bahlburg, A. A. Ehrhardt, T. M. Exner, et al., Sexual Risk Behavior Assessment Schedule-Adult-IDU Men, Baseline, (SERBAS-A-DM-1), unpublished, Columbia University, Department of Psychiatry, New York, 1988.
} 
frequency of unprotected vaginal and/or anal sex, and (d) a risk index. Abstinence indicates the participant reported not having a male or female partner for manual, oral, vaginal, or anal sex. A participant was considered monogamous if he reported only one sex partner (male or female) since his last visit (but the partner's monogamy was not assessed). Both abstinence and monogamy are dichotomous variables.

The frequency of unprotected sex is the sum of all anal or vaginal sex occasions without condom use with any sex partner. Though HIV transmission risk varies as a function of several factors, including sexual practice (anal more risky than vaginal), role (receptive more risky than insertive) and partner risk characteristics (e.g., HIV status), no corresponding adjustments were applied. This decision was based on the relative infrequency of same-sex behavior and anal sex and on the lack of relevant information (e.g., regarding partner serostatus). The number of unprotected sex occasions, having a positively skewed distribution, were categorized as follows: $0=$ no unprotected occasions, $1=$ once a month or less, 2 = two-three times per month, $3=$ once a week, $4=$ twice a week, $5=$ three time per week, $6=$ four-six times per week, $7=$ daily or more.

The risk index is a modification of indices used and validated against seroconversion rates in the Chicago MACS study $(62,63)$. Our index combines sexual practices with partner numbers as follows: $0=$ abstinent, $1=$ monogamous and uses a condom for each occasion of vaginal and/or anal sex or does not engage in vaginal or anal sex or nonmonogamous and does not engage in vaginal or anal sex, 2 = monogamous and has unprotected vaginal or anal sex or nonmonogamous and uses a condom for each occasion of vaginal and/or anal sex, $3=$ nonmonogamous and has unprotected vaginal or anal sex.

At the beginning of the study, 21 additional IDUs (16 men and 5 women) were recruited to take part in a test-retest SERBAS reliability study. The reliability of the four sexual risk behavior variables outlined above was assessed by calculating kappas (for categorical variables) and Pearson correlation coefficients (for continuous variables). Abstinence showed 100\% test-retest concordance. Agreement regarding monogamy was reported by 18 of the 20 sexually active participants $($ kappa $=.80)$. There was perfect agreement on the risk index score for 19 of the 21 participants $(r=.92)$. The variable frequency of unprotected sex also showed good test-retest reliability $(r=.78)$.

The issue of disease progression only applies to the HIV+ IDUs. HIV disease affects infected individuals in a wide variety of ways. Due to the scope of this research project, hundreds of variables were available from several domains: medical, immunological, neurological, neuropsychological, and psychosocial. The current paper is not meant to be a comprehensive evaluation of any of these 
domains. We selected a limited number of key variables based on data published from this cohort and on consultation with other HIV Center investigators. The variables selected are CD4 level, medical symptoms, Kurtzke neurological disability scale, Selective Reminding Neuropsychological Test, and the SCL-90 depression subscale.

The CD4 and HIV-related symptoms are commonly used indicators of disease progression. Blood was drawn at each visit for HIV antibody tests, CD4 counts, and various other assays. The CD4 count was included in analyses as a trichotomous variable: above 500/200-500/below 200. In addition, a research nurse collected medical histories, and physicians, blind to the participant's HIV status, conducted physical exams at every visit. The current researchers, with consultation from an HIV Center medical staff member, selected eight HIV-related symptoms or diagnoses (hereafter referred to as symptoms) that were considered to cause particular discomfort and possibly reduce the likelihood of engaging in sexual activity. These were pneumonia, diarrhea, night sweats, fever, meningitis, severe weight loss (10\% in last 3 months), shortness of breath, and persistent cough. The time frame for these symptoms was the previous 3 months, with the exception of meningitis, which covered the previous 6 months. From these symptoms, a trichotomous variable was calculated (hereafter referred to as medical symptoms): no symptoms/1 symptom/2 or more symptoms.

Participants underwent a neurological exam at each visit. Several neurological functions were assessed (pyramidal, cerebellar, brain stem, sensory, bowel/bladder, visual, mental, peripheral nerves, and extrapyramidal). The examining neurologist then classified the participant using a 10-point modified Kurtzke disability status scale (64). As in Stern et al.'s study (43), we have included the Kurtzke as a dichotomous score for which $0=$ minimal or no disability and $1=$ moderateto-severe disability ( 3 or more on the original 10-point scale). The Kurtzke neurological disability scale was chosen for our analyses since it represents a summary of neurological functioning across several domains.

The neuropsychological test chosen for our analyses was the Selective Reminding Test (65), a memory test. This test was selected since memory was the neuropsychological domain most associated with HIV disease (indicated by CD4 level) in a published report on our cohort (43). Since there is typically a learning effect over time for such memory tests, this learning effect was minimized by adjusting the participants' scores using the estimates for intercept (mean at baseline), time, age, and education from analyses involving the HIV-participants (a "nonclinical" sample). These scores were maintained as continuous scores.

Finally, the depression subscale of the SCL-90 (Symptom Check List; 66) was included as a measure of psychiatric functioning. Although the current litera- 
ture is inconsistent, depression has been associated with HIV disease in several studies (see Introduction). The score was included in analyses as a continuous variable.

\section{Data Analysis}

Analyses of the longitudinal data were performed by applying generalized estimating equations (GEEs) to regression analyses with repeated measures. The statistical method (67) takes into account the multiple visits per subject and the fact that the repeated measures from a single individual over time are likely to be correlated with one another. This method allows for missing data (a distinct advantage given the attrition in our sample). It uses all available data from all participants, and the repeated measures for each subject (up to 8 visits per variable) are treated as a cluster. Visits with low participant numbers simply contributed less to the overall results than visits involving most participants.

Another advantage of this method is its flexibility in specifying the type of regression analysis based on the distribution of the dependent variable. For the binary outcome variables, abstinence and monogamy, logistic regression analyses were used that provide estimates of the odds of the occurrence of an outcome dependent on the values of the covariates. Linear regression was applied to the analyses involving the risk index, and the regression coefficient indicates the linear association between the outcome and the covariate in the model. The categorized variable, frequency of unprotected sex, had a skewed distribution, and exponential regression was used for these analyses. The mean of this dependent variable is linked to independent variables through a logarithmic transformation so that the regression coefficient indicates the relative change in the log-transformed mean score for a unit change in the independent variables. A formulaic expression of this regression would be: $\log (\operatorname{mean}(Y))=$ linear combination of independent variables.

In the initial analyses involving the entire sample, our models were primarily designed to test for risk behavior changes over time, the effect of HIV status, and HIV status by time interaction, that is, differences in rates of change over time between HIV + and HIV - men. The demographic variables of age and education were initially included in all models as control variables. The demographic variables and the HIV status by time interaction were dropped from the model if they did not show at least a statistical trend toward an association with the dependent variable $(p<.10)$. HIV status was coded as 0 for HIV - participants and 1 for HIV+ participants. Time was entered as the number of years 
since baseline assessment. Age and education were obtained from the baseline assessment, and age was centered at its mean.

Subsequent analyses were limited to the HIV + participants and assessed the relationship between sexual risk behavior and several indicators of disease progression. Demographic variables were handled as in the previous analyses. Time was included as a covariate in all analyses since the initial analyses involving the total sample had shown changes over time for most of our outcome variables. As there are many possible nonmedical reasons why these changes over time occurred, including increasing exposure to HIV/AIDS information, time was included as a surrogate for such nonmedical effects.

\section{RESULTS}

\section{Sample Description}

The sample $(N=144)$ was $74 \%$ black/African-American, $16 \%$ Latino $(12 \%$ white, $4 \%$ black), and $10 \%$ white. At study entry, mean age was 39.7 years (range $=25-57$ ), and the men had 11.4 years of education on average. HIV+ and HIV - participants did not differ on any of these demographic variables.

Attrition was a concern since a substantial number of participants did not complete all 8 visits (see Table 1). Approximately $40 \%$ of the baseline sample were not involved by visit 7 (data collection ended in the middle of visit 8 , so even fewer participants contributed data for that visit). Some of the attrition was

Table 1. Number of Participants at Each

Semiannual Visit

\begin{tabular}{lcc}
\hline & \multicolumn{2}{c}{ Male IDUs } \\
\cline { 2 - 3 } Visit & HIV+ & HIV- \\
\hline 1 & 82 & 60 \\
2 & 64 & 48 \\
3 & 64 & 42 \\
4 & 58 & 40 \\
5 & 53 & 41 \\
6 & 46 & 36 \\
7 & 45 & 37 \\
8 & 24 & 22 \\
Total & $84^{\text {a }}$ & 60 \\
\hline
\end{tabular}

${ }^{a}$ The psychosexual interviews of two men were judged unreliable at visit 1 . 
due to AIDS-related deaths, but the proportion of HIV + participants lost to follow-up was only slightly higher than the proportion of HIV - participants. Participants assessed at visit 7 were compared to the remainder of the baseline sample on demographics, sexual risk behavior variables, and indicators of disease progression, and no significant or meaningful differences were found.

Substance use was very common among this cohort. At baseline, $80 \%$ of the sexually active men reported alcohol use with sex, 93\% used drugs in conjunction with sex (most commonly methadone, heroin, and cocaine), and 77\% reported having used more than one nonalcohol drug with sex. At baseline, all but one of the men reported some alcohol or drug use in the previous 6 months (not limited to use during sex); however, the proportion of the sample reporting any substance use during the preceding 6 months declined over time and was reduced to $80 \%$ by visit 7 (similar proportions were found when those lost to attrition were eliminated throughout).

\section{Sexual Risk Behavior as a Function of Time and HIV Status}

Figure 1 shows trends across time for each of our sexual risk behavior variables, separated by HIV status. Due to attrition, trends seen in these graphs in later visits involve substantially fewer participants than at baseline. Since final visits involve fewer observations, they contribute less data to the analyses than earlier visits. Although not included in statistical analyses, for descriptive purposes Fig. 2 shows the percentage of participants who engaged in at least one occasion of unprotected vaginal or anal sex in the 6 months prior to each visit. It can be seen that a substantial proportion of the sample continued to engage in unprotected sex. Among the HIV-participants, $95 \%$ of the men reported having unprotected sex at some point in the study. Among the HIV+ participants, $73 \%$ of the men had unprotected sex during the study.

The first set of analyses, shown in Table 2, assesses the association of the sexual risk variables with demographics (age and education), time trends, HIV status differences, and HIV status by time interactions. The demographic and interaction variables were retained in the final model if they showed at least a marginal association $(p<.10)$ with the dependent variable on an analysis-byanalysis basis; otherwise, they were removed.

Age and education were unrelated to each of the dependent variables and therefore do not appear in Table 2. There was a decrease in risk for each of our variables for both HIV + and HIV - men. HIV + men were significantly more likely to be abstinent, had lower frequencies of unprotected sex, and had lower 
DOLEZAL ET AL.

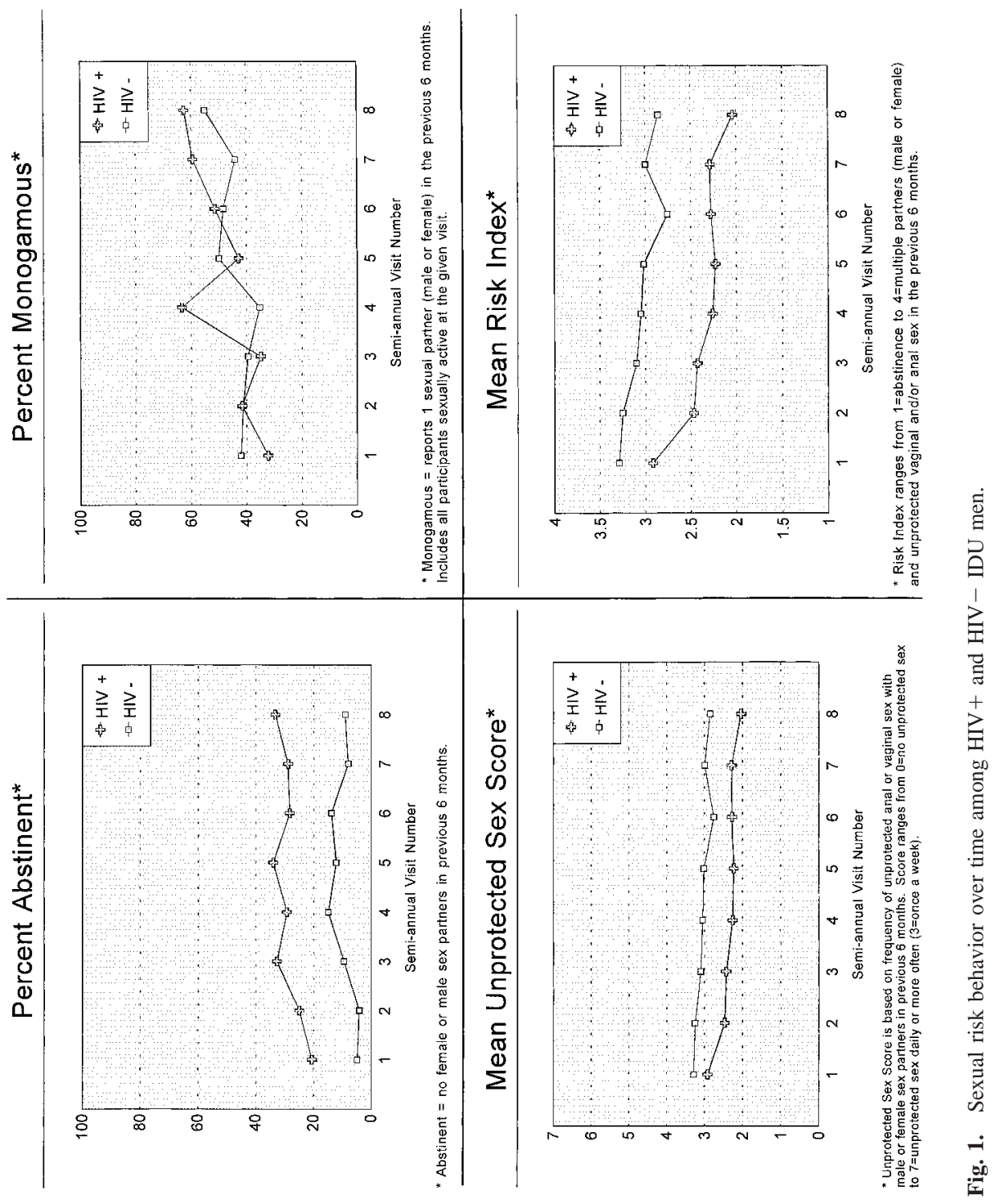




\section{Percent Engaging in Unprotected Sex*}

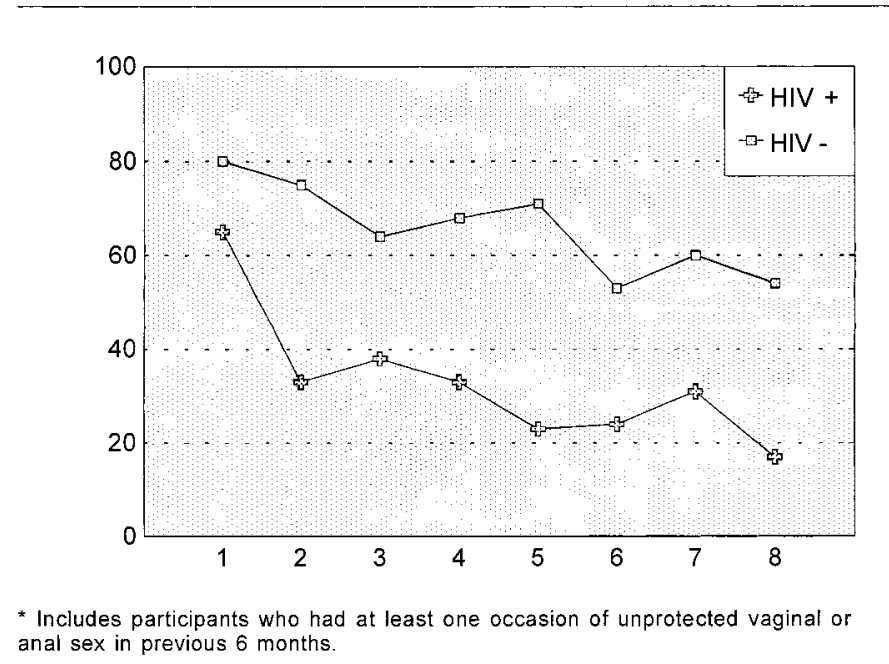

Fig. 2. Percent of HIV + and HIV - IDU men engaging in unprotected sex over time.

risk index scores than HIV-men. There was no difference in the rates of monogamy between the HIV status groups. The significant HIV status by time interaction term indicates that the HIV + men had a sharper decline in the frequency of unprotected sex than did the HIV-men.

As was noted above, the proportion of the sample reporting substance use

Table 2. HIV Status Effects and Time Trends in Sexual Risk Behavior Among Male IDUs $(N=144)$

\begin{tabular}{|c|c|c|c|c|}
\hline & $\begin{array}{c}\text { Abstinence } \\
\text { Odds Ratio }(\mathrm{CI})^{\mathrm{c}}\end{array}$ & $\begin{array}{c}\text { Monogamy } \\
\text { Odds Ratio }(\mathrm{CI})^{\mathrm{c}}\end{array}$ & $\begin{array}{c}\text { Frequency of } \\
\text { unprotected sex } \\
\text { B (SE) }\end{array}$ & $\begin{array}{l}\text { Risk index } \\
\quad \mathrm{B}(\mathrm{SE})\end{array}$ \\
\hline $\mathrm{HIV}+$ versus $\mathrm{HIV}-{ }^{\mathrm{d}}$ & $0.25(0.12-0.56)^{* * * *}$ & $1.11(0.63-1.94)$ & $-0.44(0.16)^{* *}$ & $-0.66(0.14)^{* * * *}$ \\
\hline Time & $0.84(0.71-0.99)^{*}$ & $1.25(1.06-1.49)^{* *}$ & $-0.17(0.06)^{* *}$ & $-0.18(0.04)^{* * *}$ \\
\hline HIV by time interaction ${ }^{e}$ & & & $-0.29(0.13)^{*}$ & \\
\hline
\end{tabular}

${ }^{\mathrm{a}} 0=$ abstinent; $1=$ sexually active.

${ }^{\mathrm{b}} 1=$ monogamous; $0=$ multiple partners.

${ }^{\mathrm{c}} \mathrm{CI}=95 \%$ confidence interval; $\mathrm{B}=$ regression coefficient estimate; $\mathrm{SE}=$ standard error of the estimate.

${ }^{\mathrm{d}} 0=\mathrm{HIV}-, 1=\mathrm{HIV}+$.

${ }^{\mathrm{e}}$ Age, education, and the HIV status by time interaction were assessed for all dependent variables, but were dropped from the model if not marginally associated $(p \leq .10)$.

$*=p \leq .05 ; * *=p \leq .01 ; * * *=p \leq .001$. 
declined over time. Since this decline parallels the reductions in risk behavior that were found, and since substance use has been associated with sexual risk behavior (particularly the number of sexual partners) among IDUs in other studies (68-70), it seemed possible that the decline in substance use could explain the reductions in sexual risk behavior. Therefore, the analyses assessing time and HIV status effects were redone including as a covariate a variable indicating whether substance use was reported at the given visit (results not shown). Although this covariate was significantly associated with each of the sexual risk variables except monogamy, the time trends found in the initial analyses were largely maintained. After adjusting for substance use, time effects remained significant for the risk index and monogamy, dropped to marginally significant for the frequency of unprotected sex, and were reduced to nonsignificance for abstinence.

\section{Sexual Risk Behavior as a Function of Disease Progression Among HIV + Men}

The second set of analyses is limited to HIV + participants and involves various potential indicators of disease progression. Figure 3 shows sample characteristics in terms of CD4, medical symptoms, neurological impairment, and depression scores. Memory scores are not shown since, having been adjusted to attenuate a learning effect, the scores are not comparable to Selective Reminding Test scores reported in other studies.

Results are shown in Table 3. In analyses involving CD4, the reference category is a CD4 level above 500. Compared to that group at each visit, having a CD4 level below 200 was associated with higher rates of abstinence and monogamy, and there was a trend for lower CD4 levels to be related to a lower risk index score. CD4 level was not related to the frequency of unprotected sex. Those with CD4 levels between 200 and 500 did not significantly differ from those with CD4 levels above 500 on any of the sexual risk behavior variables.

For the medical symptoms variable, observations involving no symptoms were the reference category. Compared to that group, having more than one symptom was related to more abstinence, less unprotected sex, and lower risk index scores, but not monogamy. Those with only one symptom also had less unprotected sex and lower risk index scores than the asymptomatic.

The Kurtzke neurological assessment score and the depression score were not associated with any of the dependent variables. Lower scores on the Selective Reminding Test were associated with abstinence and lower risk index scores. 


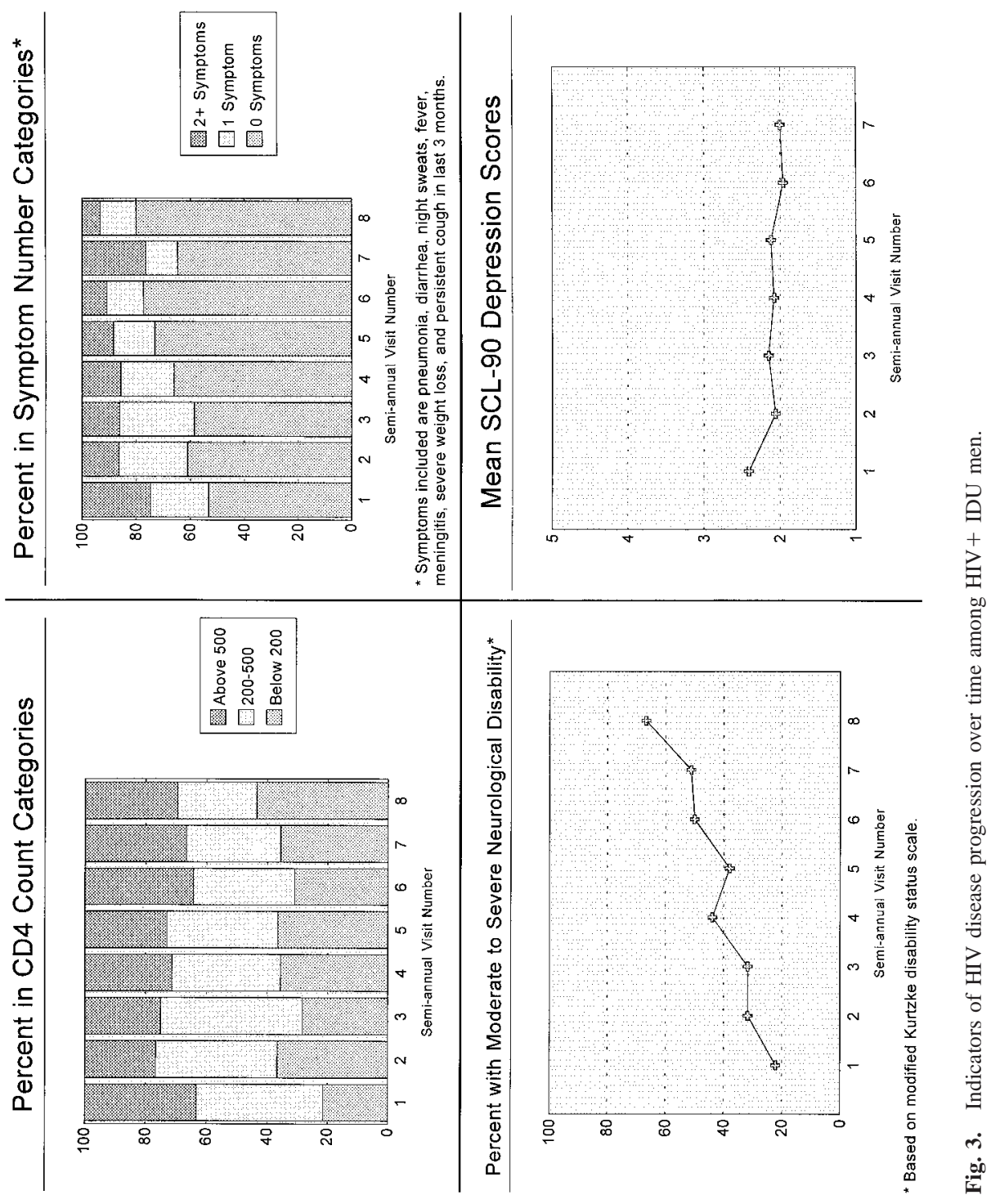


Table 3. The Association Between Sexual Risk Behavior and Indicators of HIV Disease Progression Among HIV+ IDU Men $(N=84)$

\begin{tabular}{|c|c|c|c|c|}
\hline & $\begin{array}{c}\text { Abstinence }^{\mathrm{a}} \\
\text { odds ratio }(\mathrm{CI})^{\mathrm{c}}\end{array}$ & $\begin{array}{c}\text { Monogamy } \\
\text { odds ratio }(\mathrm{CI})^{\mathrm{c}}\end{array}$ & $\begin{array}{c}\text { Frequency of } \\
\text { unprotected sex } \\
\text { B }(\mathrm{SE})^{\mathrm{c}}\end{array}$ & $\begin{array}{l}\text { Risk index } \\
\text { B }(\mathrm{SE})^{\mathrm{c}}\end{array}$ \\
\hline \multicolumn{5}{|l|}{ 1. CD4 } \\
\hline $\mathrm{CD} 4>500$ & 1 & 1 & & \\
\hline CD4 200-500 & $0.58(0.27-1.26)$ & $1.71(0.78-3.73)$ & $0.08(0.29)$ & $-0.28(0.18)$ \\
\hline $\mathrm{CD} 4<200$ & $0.37(0.14-0.97)^{*}$ & $2.66(1.04-6.80)^{*}$ & $0.36(0.32)$ & $-0.45(0.24)^{\wedge}$ \\
\hline Time & $0.90(0.74-1.10)$ & $1.42(1.08-1.86)^{*}$ & $-0.50(0.11)^{* * *}$ & $-0.19(0.05)^{* * *}$ \\
\hline $\operatorname{Age}^{\mathrm{d}}$ & & & $0.04(0.02)^{*}$ & \\
\hline \multicolumn{5}{|l|}{ 2. Symptoms } \\
\hline 0 Symptoms & 1 & 1 & & \\
\hline 1 Symptom & $0.67(0.35-1.27)$ & $1.55(0.81-2.96)$ & $-0.67(0.30)^{*}$ & $-0.35(0.16)^{*}$ \\
\hline$>1$ Symptom & $0.23(0.12-0.44)^{* * *}$ & $1.47(0.55-3.97)$ & $-1.56(0.41)^{* * *}$ & $-0.88(0.18)^{* * *}$ \\
\hline Time & $0.75(0.59-0.94)^{*}$ & $1.50(1.14-1.98) * *$ & $-0.58(0.14)^{* * * *}$ & $-0.27(0.06)^{* * *}$ \\
\hline \multicolumn{5}{|l|}{ 3. Neurological } \\
\hline Kurtzke & $0.72(0.39-1.35)$ & $0.88(0.49-1.61)$ & $-0.26(0.26)$ & $-0.15(0.16)$ \\
\hline Time & $0.93(0.75-1.15)$ & $1.47(1.10-1.95)^{* *}$ & $-0.41(0.12)^{* * * *}$ & $-0.18(0.05)^{* * *}$ \\
\hline \multicolumn{5}{|c|}{ 4. Neuropsychological } \\
\hline Memory & $1.07(1.01-1.12)^{*}$ & $0.97(0.94-1.00)^{\wedge}$ & $-0.01(0.02)$ & $0.03(0.01)^{*}$ \\
\hline Time & $0.90(0.71-1.13)$ & $1.44(1.11-1.88)^{* * *}$ & $-0.47(0.11)$ & $-0.19(0.05)^{* * *}$ \\
\hline \multicolumn{5}{|l|}{ 5. Psychosocial } \\
\hline Depression & $0.75(0.49-1.16)$ & $0.77(0.48-1.22)$ & $-0.21(0.21)$ & $-0.13(0.11)$ \\
\hline Time & $0.84(0.68-1.04)^{\wedge}$ & $1.42(1.08-1.86)^{*}$ & $-0.41(0.14) * *$ & $-0.22(0.06) * * *$ \\
\hline
\end{tabular}

${ }^{\text {a }} 0=$ abstinent; $1=$ sexually active.

b $1=$ monogamous; $0=$ multiple partners.

${ }^{\mathrm{c}} \mathrm{CI}=95 \%$ confidence interval; $\mathrm{B}=$ regression coefficient estimate; $\mathrm{SE}=$ standard error of the estimate.

${ }^{d}$ Age and education assessed for all dependent variables, but were dropped from the model if not marginally associated $(p \leq .10)$.

$\wedge=p \leq .10 ; *=p \leq .05 ; * *=p \leq .01 ; * * *=p \leq .001$.

Subsequent analyses (not shown) were conducted to see whether the association between the neuropsychological test and sexual risk behavior was maintained when controlling for medical indicators of disease progression. The Selective Reminding Test remained significantly associated with abstinence and the risk index in separate analyses, controlling for CD4 or medical symptoms.

\section{DISCUSSION}

\section{Sexual Risk Behavior as a Function of Time and HIV Status}

The study shows a continual reduction in sexual risk behavior among these IDU men throughout the 4 years of the study. For both HIV + and HIV - men, 
there were increases in abstinence and monogamy, a decrease in the frequency of unprotected sex, and a decrease in sexual risk index scores. Other studies have shown longitudinal changes in injection behavior (see Introduction), and our results suggest that IDUs have also modified their sexual risk behavior in response to the AIDS epidemic. While other studies have reported sexual behavior change in response to participation in HIV-related interventions (19), it is important to note that the changes noted in this study took place in the absence of any systematic intervention. Furthermore, they are changes that were maintained and continued over time, as opposed to short-term modifications of behavior with a return to riskier practices. Also of importance is the range of behavior change. Reductions in sexual risk behavior were found across multiple indicators. The men were more likely to abstain from sex, to limit their sexual behavior to one partner, and to reduce the number of times they had unprotected sex. These changes may be due to factors suggested above: continued exposure to AIDS information, self-monitoring as a consequence of participating in an AIDS study and being asked about sexual behavior, and increased perception of risk as acquaintances, friends, or lovers become HIV positive or even die of AIDS (by $1990,77 \%$ of the IDU men in our study knew someone who had AIDS or had died of AIDS).

While these trends are encouraging, there remains a substantial amount of sexual risk behavior in this sample. Over half of the HIV - and roughly a third of the HIV + men reported unprotected sex at any given point in the study, and consistent condom use through the entire study was rare, especially among the HIV - men. On average, HIV + men had unprotected sex 2-3 times per month, and the HIV - men did so weekly. So, although modifications in behavior are in the desired direction, sexual activity continues to put these men and their sexual partners at risk for HIV infection.

Lower rates of sexual risk behavior among HIV+ participants compared to HIV - participants indicate that HIV + IDUs had likely modified their sexual behavior prior to study entry, presumably in response to learning of their infection. HIV + men were more likely than HIV - men to be abstinent, have fewer occasions of unprotected sex, and have lower risk index scores. The general lack of significant HIV status by time interaction terms indicates that there were differences between HIV + and HIV - men early in the study, and those differences were maintained as both groups gradually continued to reduce their sexual risk behavior. An exception was the frequency of unprotected sex, for which the HIV + men had a sharper rate of decline. Monogamy was the only sexual risk variable for which there were no HIV status differences. For those reporting only one partner at visit $7(n=34)$, all partners were female, and $88 \%$ of the men identified her as a wife, lover, or steady partner (4 men referred to her as an 
acquaintance). The men apparently are increasingly involved in primary relationships to the exclusion of additional partners, possibly to reduce personal risk (to self or the primary partner if HIV-) or to prevent transmitting the virus to others (if $\mathrm{HIV}+$ ).

It is interesting to note that substance use also declined throughout the course of the study. Alcohol and drugs are linked to sexual behavior in various ways, but the associations are not likely simple or linear. Substances have varying effects that can both enhance and interfere with sexual drive and functioning (71). When heavy use of multiple drugs is common in a sample, the effects of the substances on sexual behavior are complex and multidirectional. In any case, although substance use was associated with higher levels of sexual risk behavior, most of the time trends remained independently significant. Therefore, other factors in addition to substance use contributed to reductions in sexual risk behavior.

\section{Sexual Risk Behavior as a Function of Disease Progression Among HIV + Men}

Several factors, not necessarily mutually exclusive, may contribute to the lower rates of risk behavior among HIV + participants compared to HIV - participants. It may reflect a conscientious attempt by the HIV + to prevent transmitting the virus to sexual partners. But in addition, illness appears to account for a certain amount of behavior change as well among the men. A CD4 level below 200 was associated with increased abstinence and monogamy and a lower risk index score. Similarly, the presence of HIV-related medical symptoms was also associated with increased abstinence, less unprotected sex, and lower risk index scores. Impaired performance on the Selective Reminding Test was also related to abstinence and the risk index. Since the lowest category on the risk index is abstinence, abstinence likely accounts for a significant proportion of the association with the risk index. These findings indicate that HIV + IDU men change their sexual behavior as a result of increased illness and symptomatology.

The generalizability of the findings to other IDU populations is limited by several factors. First, the typical participant is a 40-year-old African-American. These men may have quite different patterns of sexual risk behavior than, for example, Latino IDUs in Puerto Rico or younger, white IDUs in Seattle, Washington. Second, the sample is made up of volunteers recruited from public health facilities, with a minority from a methadone clinic. Men who were willing to volunteer for a research project or who are in drug treatment may not be comparable to other segments of the IDU population. Third, this sample was recruited 
in New York City. As an epicenter for the epidemic, with over half of the city's IDUs estimated to be HIV positive, it is possible that AIDS is more salient to these IDUs, compared to other cities, to the extent that there may be more outreach programs and more acquaintances affected by the disease. However, Des Jarlais did not find seroprevalence rates to be associated with levels of risk behavior when comparing several cities worldwide (72). Fourth, there were high levels of attrition in our sample. Although those lost to follow-up did not differ from the remainder of the sample at baseline in terms of demographics, sexual risk behavior, or indicators of disease progression, this does not guarantee that the same longitudinal patterns would have been found if it had been possible to follow all men throughout the 4 years of the study. Finally, although this was not an intervention study, participation in this study probably affected the participants' knowledge and behavior. Sex interviews would likely raise participants' selfawareness of their sexual risk behavior. Also, the lengthy assessments every 6 months were reminders that they were part of an AIDS study, possibly increasing their curiosity about AIDS-related information and causing them to become more educated about the disease.

In conclusion, the results suggest that IDUs, both HIV + and HIV - in New York City have reduced their sexual risk behavior through increased abstinence and monogamy and a reduction in unprotected sex. This is true even though the participants were not part of an AIDS prevention intervention in conjunction with this study.

There are also indications that being HIV positive leads to reductions in sexual risk behavior among IDUs. This is seen in the HIV status contrasts, as well as in the analyses involving HIV disease indicators, for which low CD4 levels, the presence of HIV-related symptoms, and neuropsychological impairment were associated with lower sexual risk among the HIV + men. Nevertheless, many of both the HIV + and the HIV - men continue to have unprotected sex. So, although the men in this cohort exhibit a capacity for modifying their sexual behavior, further reductions in risk behavior are necessary to minimize HIV transmission, indicating that intervention continues to be important for both HIV + and HIV - IDU men.

\section{ACKNOWLEDGMENTS}

Some of the results of this study were presented at the 21st Annual Meeting of the International Academy of Sex Research, Provincetown, Massachusetts, 1995. 
This research was supported by center grants 5-P50-MH43520 from the National Institute of Mental Health/National Institute on Drug Abuse (NIMH/ NIDA) and 2-P50-MH43520, Anke A. Ehrhardt, principal investigator, and MH30906 from NIMH.

The authors wish to thank the men who agreed to participate in this study. We also wish to thank Robert Kertzner, M.D., for his input regarding the selection of medical symptoms and Dinah Gay for library assistance.

\section{REFERENCES}

1. New York City Department of Health, AIDS Surveillance Update, Second Quarter 1996, Author, New York, 1996.

2. Anderson, W., The New York needle trial: The politics of public health in the age of AIDS, in AIDS and Contemporary History (V. Berridge and P. Strong, Eds.), Cambridge University Press, Cambridge, England, 1993, pp. 157-181.

3. Friedman, S. R., Rosenblum, A., Goldsmith, D., et al., Risk factors for HIV-1 infection among street-recruited intravenous drug users in New York City, poster session presented at the Fifth International AIDS Conference, Montreal, Canada, June 1989.

4. Battjes, R. J., and Pickens, R., HIV infection among intravenous drug abusers (IVDAs) in five U.S. cities, poster session presented at the Fourth International AIDS Conference, Stockholm, Sweden, June 1988.

5. Kleinman, P. H., Friedman, S. R., Goldsmith, D. S., et al., HIV seropositivity in a population of street intravenous drug users in New York City in 1987, poster session presented at the Fourth International AIDS Conference, Stockholm, Sweden, June 1988.

6. Yee, K., Brown, L. S., Primm, B. J., et al., Needle-sharing patterns as a predictor of HIV seroprevalence among New York City intravenous drug users (IVDUs), poster session presented at the Fifth International AIDS Conference, Montreal, Canada, June 1989.

7. Schoenbaum, E. E., Selwyn, P. A., Hartel, D., et al., HIV infection in intravenous drug users in New York City: The relation of drug use and heterosexual behaviors and race/ethnicity, poster session presented at the Fourth International AIDS Conference, Stockholm, Sweden, June 1988.

8. Nelson A. Rockefeller Institute of Government, The 1994 New York State Statistical Yearbook, Author, New York, 1994.

9. Clatts, M. C., Davis, W. R., Deren, S., et al., AIDS risk behavior among drug injectors in New York City: Critical gaps in prevention policy, in Global AIDS Policy (D. Feldman, Ed.), Bergin and Garvey, Westport, Connecticut, 1994, pp. 215-235.

10. Friedman, S. R., Des Jarlais, D. C., Sotheran, J. L., et al., AIDS and self-organization among intravenous drug users, Int. J. Addict. 22:201-219 (1987).

11. Iguchi, M. Y., Bux, D. A., Jr., Lidz, V., et al., Changes in HIV risk behavior among injecting drug users: The impact of 21 versus 90 days of methadone detoxification, AIDS, 10:1719-1728 (1996).

12. Camacho, L. M., Bartholomew, N. G., Joe, G. W., et al., Gender, cocaine and during-treatment HIV risk reduction among injection opioid users in methadone maintenance, Drug Alcohol Depend. 41:1-7 (1996).

13. Selwyn, P. A., Feiner, C., Cox, C. P., et al., Knowledge about AIDS and high-risk behavior among intravenous drug users in New York City. AIDS 1:247-254 (1987). 
14. Ronald, P. J. M., Robertson, J. R., and Roberts, J. J. K., Risk-taking behavior on the decline in intravenous drug users, Br. J. Addict. 87:115-116 (1992).

15. Celentano, D. D., Muñoz, A., Cohn, S., et al., Drug-related behavior change for HIV transmission among American injection drug users, Addiction 89:1309-1317 (1994).

16. van Ameijden, E. J. C., van den Hoek, A. A. R., and Coutinho, R. A., Injecting risk behavior among drug users in Amsterdam, 1986 to 1992, and its relationship to AIDS prevention programs, Am. J. Public Health 84:275-281 (1994).

17. Peters, A. D., Reid, M. M., and Griffin, S. G., Edinburgh drug users: Are they injecting and sharing less? AIDS 8:521-528 (1994).

18. Saxon, A. J., Calsyn, D. A., and Jackson, T. R., Longitudinal changes in injection behaviors in a cohort of injection drug users, Addiction 89:191-202 (1994).

19. Des Jarlais, D. C., Friedman, S. R., Choopanya, K., et al., International epidemiology of HIV and AIDS among injecting drug users, AIDS 6:1053-1068 (1992).

20. Feucht, T. E., Stephens, R. C., and Roman, S. W., The sexual behavior of intravenous drug users: Assessing the risk of sexual transmission of HIV, J. Drug Issues 20:195-213 (1990).

21. McDonald, C., Loxley, W., and Marsh, A., A bridge too near? Injecting drug users' sexual behavior, AIDS Care 6:317-326 (1994).

22. Booth, R. E., and Watters, J. K., How effective are risk-reduction interventions targeting injecting drug users? AIDS 8:1515-1524 (1994).

23. Rietmeijer, C. A., Kane, M. S., Simons, P. Z., et al., Increasing use of bleach and condoms among injecting drug users in Denver: Outcome of a targeted community-level HIV prevention program, AIDS 10:291-298 (1996).

24. Deren, S., Rees, D. W., Beardsley, M., et al., Outcomes of a risk-reduction intervention with high-risk populations: The Harlem AIDS project, AIDS Educ. Prev. 7:379-390 (1995).

25. Des Jarlais, D. C., Friedman, S. R., Friedmann, P., et al., HIV/AIDS-related behavior change among injecting drug users in different national settings, AIDS 9:611-617 (1995).

26. Des Jarlais, D. C., Abdul-Quader, A., and Tross, S., The next problem: Maintenance of AIDS risk reduction among intravenous drug users, Int. J. Addict. 26:1279-1292 (1991).

27. Casadonte, P. P., Des Jarlais, D. C., Friedman, S. R., et al., Psychological and behavioral impact among intravenous drug users of learning HIV test results, Int. J. Addict. 25:409-426 (1990).

28. Davoli, M., Perucci, C. A., Abeni, D. D., et al., HIV risk-related behaviors among injection drug users in Rome: Differences between 1990 and 1992, Am. J. Public Health 85:829-832 (1995).

29. Schilling, R. F., El-Bassel, N., and Gilbert, L., Predictors of changes in sexual behavior among women on methadone, Am. J. Drug Alcohol Abuse 19:409-422 (1993).

30. Martin, J. L., Dean, L., Garcia, M., et al., The impact of AIDS on a gay community: Changes in sexual behavior, substance use, and mental health. Am. J. Community Psychol. 17:269-293 (1989).

31. Janz, N. K, and Becker, M. H., The Health Belief Model: A decade later, Health Educ. Q 11: 1-47 (1984).

32. Catania, J. A., Coates, T. J., and Kegeles, S., A test of the AIDS Risk Reduction Model: Psychosocial correlates of condom use in the AMEN cohort survey, Health Psychol. 13:548-555 (1994).

33. Friedman, S. R., Jose, B., Neaigus, A., et al., Consistent condom use in relationships between seropositive injecting drug users and sex partners who do not inject drugs, AIDS 8:357-361 (1994).

34. Vanichseni, S., Des Jarlais, D. C., Choopanya, K., et al., Condom use with primary partners among injecting drug users in Bangkok, Thailand and New York City, United States, AIDS 7: 887-891 (1993).

35. Watkins, K. E., Metzger, D., Woody, G. E., et al., Determinants of condom use among intravenous drug users, AIDS 7:719-723 (1993). 
36. Skidmore, C. A., Robertson, J. R., and Roberts, J. J., Changes in HIV risk-taking behavior in intravenous drug users: A second follow-up, Br. J. Addict. 84:695-696 (1989).

37. Rabkin, J. G., Rabkin, R., and Wagner, G., Testosterone replacement therapy in HIV illness, Gen. Hosp. Psychiatry 17:37-42 (1995).

38. Exner, T. M., Dolezal, C. L., Liu, X., et al., Sexual risk behavior in the course of HIV disease: HIV + versus HIV - gay men, poster session presented at the Twenty-First Annual Meeting of the International Academy of Sex Research, Provincetown, Massachusetts, September 1995.

39. Report of a Working Group of the American Academy of Neurology AIDS Task Force, Nomenclature and research case definitions for neurologic manifestations of human immunodeficiency virus-type 1 (HIV-1) infection, Neurology 41:778-785 (1991).

40. Budka, H., Wiley, C. A., Kleihues, P., et al., HIV-associated disease of the nervous system: review of nomenclature and proposal for neuropathology-based terminology, Brain Pathol. 1: 143-152 (1991).

41. Petty, R. K. H., Recent advances in the neurology of HIV infection, Postgrad. Med. J. 70:393403 (1994).

42. Marder, K., Liu, X., Stern, Y., et al., Risk of human immunodeficiency virus type 1-related neurologic disease in a cohort of intravenous drug users, Arch. Neurol. 52:1174-1182 (1995).

43. Stern, Y., Liu, X., Marder, K., et al., Neuropsychological changes in a prospectively followed cohort of intravenous drug users with and without HIV, Neuropsychiatry Neuropsychol. Behav. Neurol. 9:83-90 (1996).

44. Bono, G., Mauri, M., Sinforiani, E., et al., Longitudinal neuropsychological evaluations of HIVinfected intravenous drug users, Addiction 91:263-268 (1996).

45. Hestad, K., Aukrust, P., Ellertsen, B., et al., Neuropsychological deficits in HIV-1 seropositive and seronegative intravenous drug users, J. Clin. Exp. Neuropsychol. 15:732-742 (1993).

46. Egan, V. G., Crawford, J. R., Brettle, R. P., et al., The Edinburgh cohort of HIV-positive drug users: Current intellectual function is impaired, but not due to early AIDS dementia complex, AIDS 4:651-656 (1990).

47. Egan, V., Brettle, R. P., and Goodwin, G. M., The Edinburgh cohort of HIV-positive drug users: Pattern of cognitive impairment in relation to progression of disease, Br. J. Psychiatry 161: 522-531 (1992).

48. Peavy, G., Jacobs, D., Salmon, D. P., et al., Verbal memory performance of patients with human immunodeficiency virus infection: Evidence of subcortical dysfunction, J. Clin. Exp. Neuropsychol. 16:508-523 (1994).

49. Bornstein, R. A., Nasrallah, H. A., Para, M. F., et al., Change in neuropsychological performance in asymptomatic HIV infection: One-year follow-up, AIDS 7:1607-1611 (1993).

50. Lipsitz, J. D., Williams, J. B. W., Rabkin, J. G., et al., Psychopathology in male and female intravenous drug users with and without HIV infection, Am. J. Psychiatry 151:1662-1668 (1994).

51. Rabkin, J. G., Johnson, J., Lin, S.-H., et al., Psychopathology in male and female HIV-positive and negative injecting drug users: Longitudinal course over 3 years, AIDS 11:507-515 (1997).

52. Rabkin, J. G., Prevalence of psychiatric disorders in HIV illness, Int. Rev. Psychiatry 8:157166 (1996).

53. Judd, F. K., and Mijch, A. M., Depression in patients with HIV and AIDS, Aust. N.Z. J. Psychiatry 28:642-650 (1994).

54. Perry, S. W., HIV-related depression, in HIV, AIDS and the Brain (R. W. Price and S. W. Perry, Eds.), Raven Press, New York, 1994, pp. 223-238.

55. Camacho, L. M., Brown, B. S., and Simpson, D. D., Psychological dysfunction and HIV/AIDS risk behavior, J. Acquir. Immune Defic. Syndr. Hum. Retrovirol. 11:198-202 (1995).

56. Nemoto, T., Foster, K., and Brown, L., Effect of psychological factors on risk behavior of human immunodeficiency virus (HIV) infection among intravenous drug users (IVDUs), Int. J. Addict. 26:441-456 (1991). 
57. Gorman, J. M., Kertzner, R., Todak, G., et al., Multidisciplinary baseline assessment of gay men with and without HIV infection. I: Overview of study design, Arch. Gen. Psychiatry 48: 120-123 (1991).

58. Meyer-Bahlburg, H. F. L., Exner, T. M., Dellenbaugh, C., et al., Sexual identity and sexual risk behavior in intravenous drug using men, The VII International Conference on AIDS, Florence, Italy, June 16-21, 1991, Abstract Book, Vol. 1, \#M.D.4035, 398 (June 1991).

59. Meyer-Bahlburg, H. F. L., Exner, T. M., Dolezal, C. L., et al., Change of sexual risk behavior in IVDU men, The VIII International Conference on AIDS/III STD World Congress, Amsterdam, The Netherlands, July 19-24, 1992, Poster Abstracts, Vol. 2, \#PoD 5086, D401 (July 1992).

60. Centers for Disease Control, CDC classification system for human T-lymphotropic virus type III/lymphadenopathy-associated virus infections, MMWR Morb. Mortal. Wkly. Rep. 35:334339 (1986).

61. Meyer-Bahlburg, H. F. L., Exner, T. M., Lorenz, G., et al., Sexual risk behavior, sexual functioning, and HIV-disease progression in gay men, J. Sex. Res. 28:3-27 (1991).

62. Joseph, J. G., Montgomery, S., Kessler, R., et al., Two-year longitudinal study of behavioral risk reduction in a cohort of homosexual men, poster session presented at the Third International Conference on AIDS, Washington, D.C., 1987.

63. Ostrow, D. G., Risk reduction for transmission of human immunodeficiency virus in high-risk communities, Psychiatr. Med. 7:79-96 (1989).

64. Kurtzke, J. F., Rating neurologic impairment in multiple sclerosis: An expanded disability status scale (EDSS), Neurology 33:1444-1452 (1983).

65. Buschke, H., and Fuld, P., A. Evaluating storage, retention, and retrieval in disordered memory and learning, Neurology 24:1019-1025 (1974).

66. Derogatis, L. R., Lipman, R. S., and Covil, L., SCL-90: An outpatient psychiatric rating scalepreliminary report, Psychopharmacol. Bull. 9:13-28 (1973).

67. Liang, K. Y., and Zeger, S. L., Longitudinal data analysis using generalized linear models, Biometrika 73:13-22 (1986).

68. Ross, M. W., Kelaher, M., Wodak, A., et al., Predictors of intoxicated sex in injecting drug users, J. Addict. Dis. 13:69-80 (1994).

69. Longshore, D., Anglin, M. D., Hseih, S., et al., Sexual behaviors and cocaine preference among injection drug users in Los Angeles, J. Drug Issues 23:363-374 (1993).

70. McBride, D. C., Inciardi, J. A., Chitwood, D. D., et al., Crack use and correlates of use in a national population of street heroin users, J. Psychoactive Drugs 24:411-416 (1992).

71. Crenshaw, T. L., and Goldberg, J. P., Sexual Pharmacology: Drugs that Affect Sexual Functioning, W. W. Norton and Company, New York, 1996.

72. Des Jarlais, D. C., Cross-national studies of AIDS among injecting drug users. Addiction 89: 383-392 (1994). 
